\title{
INSECTICIDE RESISTANCE MANAGEMENT OF LEAFROLLERS (LEPIDOPTERA: TORTRICIDAE) IN NEW ZEALAND
}

\author{
P.L. LO ${ }^{1}$, J.T.S. WALKER ${ }^{1}$ and D.M. SUCKLING ${ }^{2}$ \\ ${ }^{1}$ HortResearch, Hawke's Bay Research Centre, Private Bag 1401, \\ Havelock North, New Zealand \\ ${ }^{2}$ HortResearch, Canterbury Research Centre, P.O. Box 51, Lincoln, Canterbury, \\ New Zealand
}

\begin{abstract}
Resistance to the organophosphate insecticide azinphos-methyl has been previously identified in two species of leafroller (Lepidoptera: Tortricidae) in New Zealand. This study confirmed resistance in a third species, Ctenopseustis obliquana. Populations of Epiphyas postvittana, Planotortrix. octo and C. obliquana resistant to azinphos-methyl were not cross-resistant to lufenuron. A separate population of $C$. obliquana was resistant to taufluvalinate. Cross-resistance between azinphosmethyl and tebufenozide occurred in $P$ octo and $C$. obliquana, but not in E. postvittana. This difference in cross-resistance between the three species suggests that at least two different detoxification mechanisms may be operating. Therefore we cannot generalise about whether resistant populations of each species will be fully susceptible to new insecticides. Lufenuron is recommended as a key insecticide within a resistance management programme for leafrollers, while tebufenozide should be used with caution.

Keywords: leafroller, Tortricidae, insecticide resistance, organophosphate, insect growth regulator.
\end{abstract}

\section{INTRODUCTION}

Resistance to pesticides is an increasing problem in New Zealand and around the world, as more pests become resistant to more products. Examples of pests in New Zealand that are resistant to herbicides, fungicides, miticides and insecticides are given by Bourdôt and Suckling (1996). Resistance can develop to virtually any crop protection product that is designed to kill pests. The likelihood of resistance occurring and the speed with which it develops depends on a combination of factors that make up the "selection pressure" (Georghiou and Taylor 1977a, b). These factors include (a) the biology and ecology of the pest, (b) how toxic and persistent a pesticide is and (c) the frequency of product use. Once a pest has developed resistance to one pesticide, it may also be "cross-resistant" to other pesticides that have the same mode of action. In rare cases, a pest can develop "multiple resistance" to more than one class of pesticide with different modes of action.

Leafrollers (Lepidoptera: Tortricidae) are key pests of fruit tree and vine crops in New Zealand. There are six common pest species (Wearing et al. 1991) but only Epiphyas postvittana (lightbrown apple moth) occurs throughout the country. Two species in three regions have developed resistance to the organophosphate insecticide azinphos-methyl (Gusathion). They are E. postvittana in Nelson (Suckling et al. 1984), and Planotortrix octo (greenheaded leafroller) in Central Otago (Wearing 1995a) and Hawke's Bay (Lo et al. 1997). Azinphos-methyl resistant E. postvittana were cross-resistant to phosmet, chlorpyrifos and carbaryl (Suckling and Khoo 1990). Azinphos-methyl resistant $P$. octo from Central Otago have cross-resistance to chlorpyrifos, carbaryl (Wearing 1995a) and to the insect growth regulator tebufenozide (Wearing 1998). In laboratory studies, Wearing (1998) showed that selection of 
resistant $P$. octo with tebufenozide or azinphos-methyl increased the level of resistance to both insecticides.

In 1996 the New Zealand pipfruit industry began implementing an integrated fruit production (IFP) programme (Walker et al. 1997), which has now been adopted by over $85 \%$ of growers. Pest management under the former "conventional" programmes was based on two-weekly sprays of organophosphate insecticides during the growing season. After several decades of such use on many orchards, it was not surprising that resistance should develop to azinphos-methyl, the main insecticide used. Since the introduction of IFP, pesticide use overall has declined dramatically, particularly organophosphates, which have been replaced by insect growth regulators. It is vital that these new insecticides are used within resistance management programmes if reliable and sustainable pest management is to be achieved.

In this paper we give an overview of the current situation with regard to management of organophosphate-resistant populations of leafrollers and update the information on resistance to two insect growth regulators (a benzoylhydrazine and a benzoylurea) and a pyrethroid insecticide.

\section{METHODS}

Areas where leafrollers were suspected of having developed resistance to insecticides were initially identified from reports that growers were having difficulty achieving adequate control of these pests. Larvae were collected from pip and berryfruit orchards (Hawke's Bay), shelter or scrub adjacent to orchards (Nelson) and an avocado orchard (Bay of Plenty). The larvae were reared on artificial diet in the laboratory to determine their species, and adults from suspected resistant species were mated to produce neonate larvae for testing with insecticides. The larvae tested were the $\mathrm{F}_{1}$ offspring of field-collected wild-wild crosses, except for the Ctenopseustis obliquana (brownheaded leafroller) from Hawke's Bay tested with azinphos-methyl. The latter were the offspring of wild moths crossed with susceptible laboratory moths.

The insecticides that each species of leafroller was tested against are given in Table 1. Full details of the procedures used are given in Wearing (1995a, 1998) and Lo et al. (1997). In each case the data from field populations were compared against fully susceptible larvae from laboratory colonies maintained by HortResearch at the Mount Albert Research Centre in Auckland. These colonies were established from susceptible leafrollers and have not been exposed to insecticides for many generations. Different bioassays were used to test different classes of insecticides. Organophosphates and the pyrethroid were tested using a Potter tower where larvae were sprayed with the insecticide before being transferred onto artificial diet (direct spray bioassay). Insect growth regulators were tested either by mixing the insecticide into artificial diet (diet incorporation) or by spraying it onto artificial diet (sprayed diet) and then introducing leafroller larvae. There were 4-6 replicates.

Each population was tested with a range of insecticide concentrations and the resulting mortalities were analysed by standard probit analysis (LeOra software 1987) to produce dose-mortality response curves and $\mathrm{LC}_{50}$ values (the concentration estimated to kill $50 \%$ of the population). By convention, resistance was defined as a five-fold or greater difference between the $\mathrm{LC}_{50}$ value of the test population and a reference susceptible strain (Suckling and Khoo 1990).

\section{RESULTS}

The results of testing three species of leafroller for insecticide resistance are summarised in Table 1. Azinphos-methyl resistant populations of P. octo in Hawke's Bay were cross-resistant to tebufenozide (Mimic), but not to chlorpyrifos (Lorsban) or lufenuron (Match). The wild-susceptible crosses of C. obliquana from the Longlands area, Hawke's Bay had a 2-6-fold resistance factor to azinphos-methyl. The resistance factor for wild-wild resistant moths would be expected to be approximately double that recorded (i.e. 4-12-fold), or higher in the case of homozygous resistance. These resistant $C$. obliquana were cross-resistant to tebufenozide, but not to lufenuron. A range of levels of resistance to the pyrethroid taufluvalinate (Mavrik) was recorded in 
C. obliquana from the Bay of Plenty. Unlike the previous two species, azinphosmethyl resistant $E$. postvittana from Nelson were not resistant to either tebufenozide or lufenuron.

TABLE 1: Susceptibility of field-collected leafrollers compared with fully susceptible laboratory strains to five insecticides tested between 1994 and 1999.

\begin{tabular}{|c|c|c|c|c|c|c|}
\hline \multirow{2}{*}{$\begin{array}{l}\text { Species (Region) } \\
\text { Insecticide (Class }{ }^{1} \text { ) }\end{array}$} & \multicolumn{2}{|c|}{$\mathrm{LC}_{50}(\mathrm{ppm})$} & \multirow{2}{*}{$\begin{array}{l}\text { Resistance } \\
\text { factor }^{2} \\
\text { ory }\end{array}$} & \multirow[t]{2}{*}{ Bioassay $^{3}$} & \multirow[t]{2}{*}{ Site } & \multirow{2}{*}{$\begin{array}{c}\text { Susceptibility } \\
\text { to azinphos } \\
\text {-methyl }\end{array}$} \\
\hline & Orchard & Labora & & & & \\
\hline \multicolumn{7}{|l|}{ P. octo (Hawke's Bay) } \\
\hline \multirow[t]{3}{*}{ azinphos-methyl (OP) } & 41.9 & 6.1 & $7^{4}$ & DS & $\mathrm{A}$ & Resistant \\
\hline & 66.5 & & $11^{4}$ & & B & Resistant \\
\hline & 194.5 & & $32^{4}$ & & $\mathrm{C}$ & Resistant \\
\hline \multirow[t]{2}{*}{ chlorpyrifos (OP) } & 50.0 & 22.6 & 2 & DS & $\mathrm{D}$ & Resistant \\
\hline & 70.4 & & 3 & & A & \\
\hline tebufenozide (BH) & 2.1 & 0.3 & 6 & DI & A & \\
\hline lufenuron (BU) & 2.8 & 1.5 & 2 & DI & A & \\
\hline \multicolumn{7}{|c|}{ C. obliquana (Hawke's Bay) } \\
\hline \multirow[t]{2}{*}{ azinphos-methyl } & $8.2-23.6$ & 4.0 & $2-6^{5}$ & DS & $\mathrm{E}$ & Resistant \\
\hline & $7.3-24.7$ & 4.0 & $2-6^{5}$ & DS & $\mathrm{F}$ & Resistant \\
\hline tebufenozide & 77.5 & 5.6 & 14 & SD & G & Resistant \\
\hline lufenuron & 5.1 & 2.7 & 2 & DI & $\mathrm{H}$ & Resistant \\
\hline \multicolumn{7}{|c|}{ C. obliquana (Bay of Plenty) } \\
\hline taufluvalinate (SP) & $8.4-47.2$ & 2.8 & $3-17$ & DS & I & Unknown \\
\hline \multicolumn{7}{|c|}{ E. postvittana (Nelson) } \\
\hline \multirow[t]{2}{*}{ tebufenozide } & 5.6 & 2.9 & 2 & DI & $\mathrm{J}$ & Susceptible \\
\hline & 9.4 & 2.9 & 3 & DI & $\mathrm{K}$ & Resistant \\
\hline lufenuron & 3.1 & 1.8 & 2 & DI & K & \\
\hline
\end{tabular}

${ }^{1} \mathrm{OP}$ organophosphate, $\mathrm{BH}$ benzoylhydrazine, BU benzoylurea, SP synthetic pyrethroid.

${ }^{2}$ Orchard $\mathrm{LC}_{50} /$ Laboratory $\mathrm{LC}_{50}$; resistance defined as a 5 fold difference between field and susceptible populations.

${ }^{3}$ DS direct spray, DI diet incorporation, SD sprayed diet.

${ }^{4}$ Data from Lo et al. 1997.

${ }^{5}$ Larvae tested were the offspring of wild moths crossed with susceptible laboratory moths, therefore the resistance factor is assumed to be half that expected for wildwild resistant moths of the same genetic mix.

\section{DISCUSSION}

Insect growth regulator insecticides that are commonly used in New Zealand for the control of leafrollers and codling moth (Cydia pomonella) are currently limited to tebufenozide and lufenuron. In this study, all three leafroller species with azinphosmethyl resistance were shown to be susceptible to lufenuron. The same applies to the Otago P. octo population (C.H. Wearing, pers. comm.). Lufenuron therefore appears to be useful for managing resistant populations of leafrollers in the Mariri-Bluffs area (Nelson), Dumbarton, (Central Otago), and the Twyford and Longlands areas of Hawke's Bay. The control of resistant leafrollers in Twyford has greatly improved since lufenuron has been widely used. In the case of E. postvittana, the tests with azinphos-methyl were done on a strain from Mariri where the resistance frequency may have been lower than ideal for this test. Lufenuron has not been tested on taufluvalinate resistant $C$. obliquana from the Bay of Plenty, so we cannot comment on its effectiveness in this case. 
The finding by Wearing (1998) that azinphos-methyl resistance in $P$. octo from Central Otago extended to tebufenozide was the first such case of cross-resistance reported. The present study confirmed that the same pattern of resistance is present in $P$. octo and $C$. obliquana from Hawke's Bay. Cross-resistance between organophosphates and tebufenozide is also suspected in a North American leafroller (Waldstein et al. 1999). The occurrence of cross-resistance to tebufenozide in P. octo and $C$. obliquana but not in E. postvittana, suggests that there are at least two different insecticide detoxification pathways operating in these species.

Overseas research illustrates the potential for further cross-resistance in New Zealand leafrollers. Sauphanor and Bouvier (1995) found cross-resistance in codling moth between different types of insect growth regulators. Furthermore, Sauphanor et al. (1998) reported on codling moth field strains with resistance to organophosphates, pyrethroids and two benzoylureas. They concluded that resistance to pyrethroids could confer cross-resistance to benzoylureas, and to a lesser extent to organophosphates. This seems likely to be due to enhanced levels of oxidative metabolism (Sauphanor et al. 1997), which was also a factor in azinphos-methyl-resistant E. postvittana (Armstrong and Suckling 1990). This mechanism could represent the key to the observed resistance spectrum with the New Zealand species, but no biochemistry has been undertaken to investigate this question.

Mating disruption has been used successfully in resistance management for both E. postvittana (Suckling et al. 1990) and P. octo (Wearing 1995b), and is likely to offer similar benefits for $C$. obliquana. However, the cost of this tactic for leafroller control has so far prevented the wider uptake necessary for proactive resistance management (i.e. before resistance or control failure is detected). The risks presented by resistance in terms of limiting product choices for leafroller control need to be weighed up by pipfruit growers against the additional short term costs of control using a solution that is more expensive, but has the potential to delay or prevent resistance development.

At present the best management strategy for resistant $P$. octo and C. obliquana is to avoid using organophosphates wherever possible, and to use lufenuron supplemented with pheromone mating disruption. For E. postvittana and susceptible populations of the other two species, tebufenozide can and should be used as well as lufenuron to preserve the susceptibility of leafrollers to both products. In practice, however, lufenuron is only registered for use on apples and cannot be applied after mid December, while the cost of pheromone dispensers and the need to control other pests means that mating disruption is not widely used. Therefore on apples the most practical strategy is to use lufenuron against the first generation of leafrollers and to use tebufenozide, which is the only effective IFP-compatible insecticide available for mid and late summer use, against later generations only if necessary.

The current resistance management options on crops other than pipfruit are more limited because lufenuron is not available. However, several new classes of insecticide with a range of different modes of action against lepidopterous pests are poised to enter the New Zealand marketplace. In the meantime, the careful use of a range of insecticides with different modes of action is important to delay any further development of resistance.

In conclusion, it is important to implement a resistance management strategy to enhance the long-term effectiveness of current and new insecticides. Such a strategy will also help to minimise the risk of resistant leafrollers spreading from their relatively small current distributions. Lufenuron is a key chemical weapon against resistant leafrollers, but tebufenozide needs to be used with caution. In future, the optimal solution depends in part on the development and registration of alternative insecticides with no cross-resistance risk. We found different patterns of insecticide resistance among the three leafroller species. Therefore we cannot generalise about whether resistant populations of each species will be fully susceptible to new insecticides. 


\section{ACKNOWLEDGEMENTS}

We thank the orchardists from whose properties leafrollers were collected, and Lyn Cole, Vanessa Murrell, Pat Sale and Peter Shaw for their technical assistance. The research was funded by ENZAFruit International and Novartis New Zealand Ltd. The manuscript was critically reviewed by Drs Andrew Tomkins, Howard Wearing and Maureen O'Callaghan.

\section{REFERENCES}

Armstrong, K.F. and Suckling, D.M., 1990. Correlation and inheritance of azinphos-methyl resistance in the lightbrown apple moth (Lepidoptera: Tortricidae). Pestic. Biochem. Physiol. 36: 281-289.

Bourdôt, G.W. and Suckling, D.M. (Eds), 1996. Pesticide Resistance: Prevention and Management. New Zealand Plant Protection Society Inc., Christchurch.

Georghiou, G.P. and Taylor, C.E., 1977a. Genetic and biological influences in the evolution of insecticide resistance. J. Econ. Entomol. 70: 319-323.

Georghiou, G.P. and Taylor, C.E., 1977b. Operational influences in the evolution of insecticide resistance. J. Econ. Entomol. 70: 653-658.

Lo, P.L., Walker, J.T.S. and Suckling, D.M., 1997. Resistance of Planotortrix octo (greenheaded leafroller) to azinphos-methyl in Hawke's Bay. Proc. 50th N.Z. Plant Prot. Conf: : 409-413.

Sauphanor, B. and Bouvier, J.C., 1995. Cross-resistance between benzoylureas and benzoylhydrazines in the codling moth, Cydia pomonella L. Pestic. Sci. 45: 369375.

Sauphanor, B., Cuany, A., Bouvier, J.C., Brouse, V., Amichot, M. and Bergé, J.B., 1997. Mechanism of resistance to deltamethrin in Cydia pomonella (L.) (Lepidoptera: Tortricidae). Pestic. Biochem. Physiol. 58: 109-117.

Sauphanor, B., Bouvier, J.C. and Brosse, V., 1998. Spectrum of insecticide resistance in Cydia pomonella (Lepidoptera: Tortricidae) in Southeastern France. J. Econ. Entomol. 91: 1225-1231.

Suckling, D.M., Chapman, R.B. and Penman, D.R., 1984. Insecticide resistance in the lightbrown apple moth (Epiphyas postvittana Walker)(Lepidoptera: Tortricidae): larval response to azinphosmethyl. J. Econ. Entomol. 77: 579-582.

Suckling, D.M. and Khoo, J.G.I., 1990. Cross resistance in the lightbrown apple moth (Lepidoptera: Tortricidae). N.Z. J. Crop Hort. Sci. 18: 173-180.

Suckling, D.M., Khoo, J.G.I. and Rogers, D.J., 1990. Resistance management of Epiphyas postvittana (Lepidoptera: Tortricidae) using mating disruption. N.Z. J. Crop Hort. Sci. 18: 89-98.

Waldstein, D.E.; Reissig, W.H.; Scott, J.G. and Straub, R.W., 1999. Susceptibility of obliquebanded leafroller (Lepidoptera: Tortricidae) populations from commercial apple orchards and an unsprayed habitat in New York to tebufenozide. J. Econ. Entomol. 92: 1251-1255.

Walker, J.T.S., Hodson, A.J., Wearing, C.H., Bradley, S.J., Shaw, P.W., Tomkins, A.R., Burnip, G.M., Stiefel, H.E. and Batchelor, T.A., 1997. Integrated fruit production for New Zealand pipfruit: evaluation of pest management in a pilot programme. Proc. 50th N.Z. Plant Prot. Conf:: 258-263.

Wearing, C.H., Thomas, W.P., Dugdale, J.S. and Danthanarayana, W., 1991. Tortricid pests of pome and stone fruits, Australian and New Zealand species. Pp 453-472 In: Tortricid Pests: Their Biology, Natural Enemies and Control, L.P.S. Geest and H.H. van der Evenhuis (Eds); Elsevier, Amsterdam.

Wearing, C.H., 1995a. Resistance of Planotortrix octo to organophosphate insecticides in Dumbarton, Central Otago. Proc. 48th N.Z. Plant Prot. Conf.: 40-45.

Wearing, C.H., 1995b. Mating disruption for management of organophosphate resistance in the greenheaded leafroller Planotortrix octo. Proc. 48th N.Z. Plant Protection Conf.: 46-51.

Wearing, C.H., 1998. Cross-resistance between azinphos-methyl and tebufenozide in the greenheaded leafroller, Planotortrix octo. Pestic. Sci. 54: 203-211. 